\title{
Téoros
}

Revue de recherche en tourisme

\section{Les concepts et les réalités du tourisme social et du tourisme solidaire en Afrique}

\section{Charles-Étienne Bélanger, Louis Jolin et Jean-Marc Mignon}

Volume 26, numéro 3, automne 2007

Tourisme et solidarité

URI : https://id.erudit.org/iderudit/1071003ar

DOI : https://doi.org/10.7202/1071003ar

Aller au sommaire du numéro

Éditeur(s)

Université du Québec à Montréal

ISSN

0712-8657 (imprimé)

1923-2705 (numérique)

Découvrir la revue

Citer cet article

Bélanger, C.-É., Jolin, L. \& Mignon, J.-M. (2007). Les concepts et les réalités du tourisme social et du tourisme solidaire en Afrique. Téoros, 26(3), 25-30.

https://doi.org/10.7202/1071003ar d'utilisation que vous pouvez consulter en ligne. 


\section{Les concepts et les réalités du tourisme social et du tourisme solidaire en Afrique}

\section{Charles-Étienne Bélanger, Louis Jolin et Jean-Marc Mignon}

Dans le secteur du tourisme, l'Afrique est l'une des régions du monde qui a connu la plus forte croissance au cours des dernières années. De 2000 à 2005, les arrivées de touristes internationaux en Afrique sont passées de 28 à 40 millions en 2005 (40,3 millions en 2006), avec une progression annuelle moyenne de 5,6\% contre 3,1\% pour le monde entier. De plus, au cours de la même période, les recettes du tourisme international de l'Afrique ont doublé, passant de 10,5 à 21,3 milliards USD, selon les chiffres de l'Organisation mondiale du tourisme (OMT) ${ }^{1}$.

Ces données, aussi impressionnantes soient-elles, ne doivent toutefois pas faire oublier la part de marché de l'Afrique à l'échelle mondiale qui reste toujours très faible avec 4,8\% des arrivées internationales en 2005.

Dans ce contexte, comme l'affirmait le secrétaire général de l'OMT, Francesco Frangialli, à l'occasion de la manifestation TourismAfrica à Genève en septembre 2006², il existe un grand potentiel pour faire en sorte que le tourisme puisse contribuer au commerce et au développement de l'Afrique tout en participant aux objectifs du Millénaire pour le développement, dont l'un des axes majeurs vise à réduire de moitié l'extrême pauvreté d'ici 2015.

C'est aussi dans ce contexte, mais de façon modeste, que le Bureau international du tourisme social (BITS) a créé une section Afrique à l'occasion de son congrès en $2004^{3}$, avec les objectifs de concourir au développement du tourisme social dans les pays africains où cela est possible et d'aider au développement du tourisme solidaire à destination de l'Afrique.

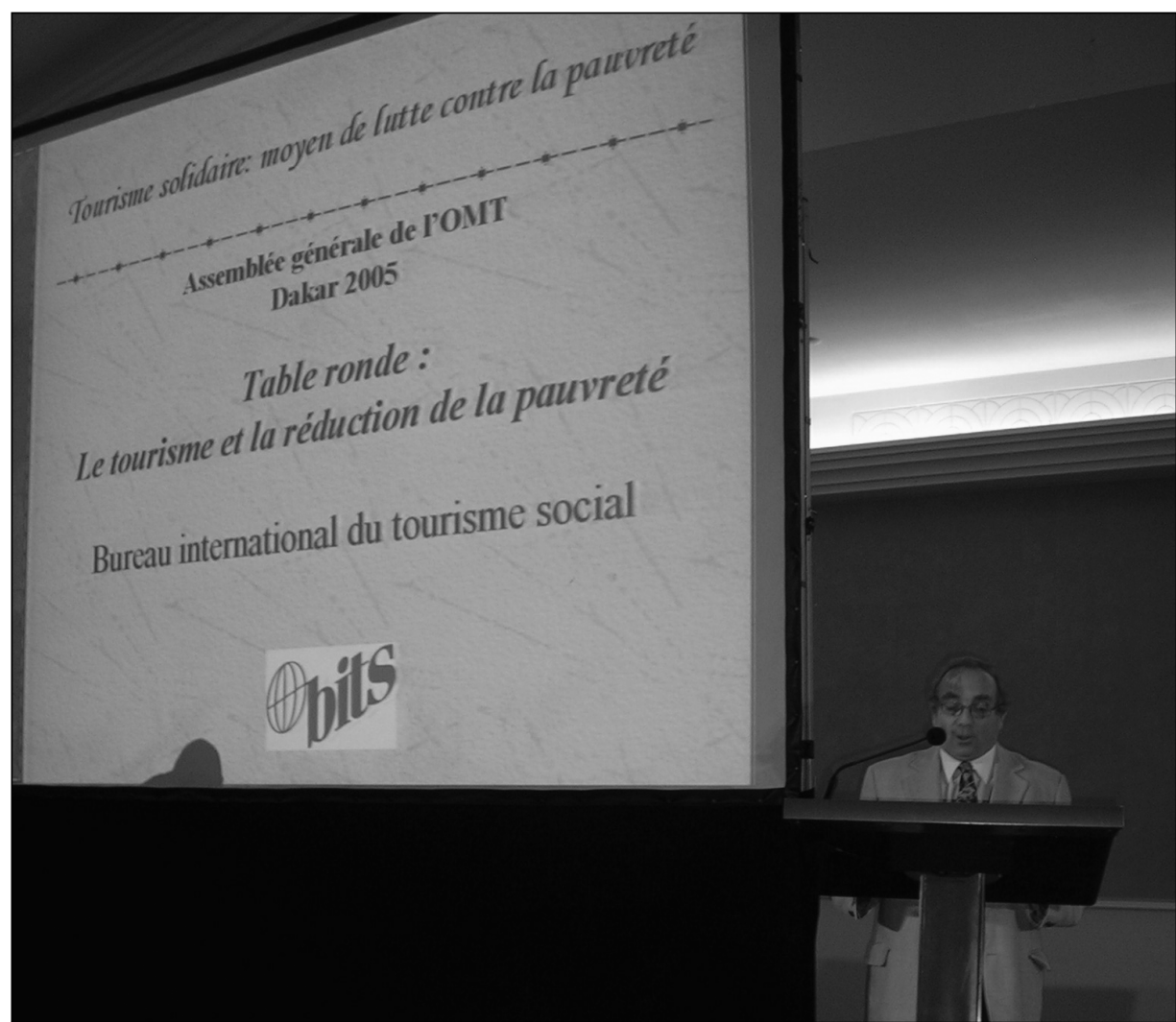

Monsieur Jean-Marc Mignon, vice-président du BITS lors de l'assemblée générale de l'OMT en 2005 à Dakar. Photo : Louis Jolin

Le tourisme social et le tourisme solidaire peuvent-ils vraiment représenter des voies appropriées pour développer le potentiel touristique de l'Afrique, là où les conditions le permettent, en complémentarité au tourisme international traditionnel ?

Le tourisme social, comme cela a pu être démontré dans d'autres régions du monde, en Europe mais aussi dans certains pays des Amériques, peut jouer un rôle significatif dans le développement et la croissance du tourisme interne grâce à la mise en place de politiques, de stratégies et d'actions per- mettant à un plus grand nombre de personnes de participer à l'activité touristique.

Le tourisme solidaire, lorsqu'il est développé selon certains critères, peut de son côté contribuer directement au développement des communautés locales et à la réduction de la pauvreté dans les pays en développement.

La connaissance des concepts et des réalités du tourisme social et du tourisme solidaire en Afrique et l'identification des actions potentielles à mener sur ce continent constituaient les objectifs d'une étude ambitieuse 
réalisée par le BITS, sous la coordination de Charles-Étienne Bélanger, de Louis Jolin et de Jean-Marc Mignon. Ce projet a été rendu possible grâce au soutien de l'Organisation mondiale du tourisme, du ministère français des Affaires étrangères et de l'Union nationale des associations de tourisme (UNAT). Le présent article vise à rendre compte des faits saillants de cette étude rendue publique en mai 2007.

\section{Aperçu méthodologique}

L'étude comprend trois parties dont chacune fait appel à une approche méthodologique distincte.

1. Une partie conceptuelle traitant des concepts de tourisme social et de tourisme solidaire dans leurs dimensions internationale et africaine. Ce travail a été réalisé par des universitaires sous la responsabilité du comité scientifique du BITS.

Un premier document d'une quinzaine de pages a été rédigé par deux membres du comité scientifique du BITS, les professeurs Louis Jolin et Mimoun Hillali ; le texte a fait l'objet de consultations auprès d'experts africains, dont les avis écrits ont été synthétisés et intégrés au document de base ${ }^{4}$.

2. Une enquête auprès des administrations nationales du tourisme (ANT) sur les rôles et les actions des pouvoirs publics en matière de tourisme social et de tourisme solidaire. Le but de l'enquête effectuée auprès des ANT en Afrique visait à recueillir des renseignements sur le tourisme interne, sur les politiques et les programmes en matière de tourisme social et sur le soutien à des projets locaux de tourisme solidaire.

La préparation et la conception du questionnaire ont été réalisées par le Secrétariat du BITS, sous la responsabilité de Charles-Étienne Bélanger, directeur du BITS à Bruxelles, en collaboration avec les services de la Commission de l'Organisation mondiale du tourisme pour l'Afrique. L'analyse des résultats a été effectuée par le Secrétariat du BITS qui a bénéficié du soutien de l'OMT pour la réalisation de cette étude.

3. Une enquête auprès d'acteurs locaux du tourisme solidaire en Afrique, principalement des organisations non gouvernementales (ONG), des coopératives et des associations. L'objectif de cette en- quête était d'identifier des expériences réussies dans le domaine du tourisme solidaire, les facteurs qui ont contribué à cette réussite et leurs impacts sur les communautés d'accueil.

La préparation du questionnaire, la collecte des données et l'analyse des résultats ont été réalisées par l'Union nationale des associations de tourisme et de plein air (UNAT) en France, sous la responsabilité de son délégué général, Jean-Marc Mignon ${ }^{5}$. L'UNAT a bénéficié du soutien du ministère français des Affaires étrangères pour la réalisation de cette enquête.

\section{Des concepts pertinents pour le continent africain}

Le premier volet de l'étude rappelle que le tourisme social ${ }^{6}$ est un concept qui est né dans les pays industrialisés, mais qu'il n'est pas inopportun de considérer son développement à l'intérieur de plusieurs pays africains.

Parmi les gouvernements des pays en développement qui ont choisi le tourisme comme levier économique, plusieurs en ont orienté le développement dans une perspective internationale, prenant en considération que les touristes étrangers apportent avec eux des devises fortes, qu'ils constituent une clientèle payante et que leurs dépenses touristiques ont un impact non négligeable sur l'économie nationale.

Par ailleurs, les conditions de vie de larges segments des populations des pays du Sud, notamment des pays africains, font en sorte que leurs priorités dans bien des cas vont à la survie et, dans les autres, à l'obtention d'un travail stable, d'un logement décent, d'une éducation suffisante...

Dans ce contexte, le tourisme social, dans sa définition traditionnelle, peut-il s'implanter et offrir une réponse aux besoins des pays africains? Ou n'est-il que l'apanage des pays industrialisés?

Le tourisme social peut avoir un sens en Afrique si nous explorons des équations susceptibles de dégager quelques repères prospectifs intéressants. L'expérience de certains pays d'Amérique latine (Mexique, Brésil, Argentine...) dans la résolution de ces équations doit être mise à profit. Cinq équations ont été considérées dans la partie conceptuelle de l'étude.
Afin de dégager des repères intéressants, les chercheurs ont mis le tourisme social en relation avec différents facteurs :

1. L'essor des classes moyennes dans plusieurs pays africains: c'est en prenant d'abord en considération la demande d'une classe moyenne nationale naissante, en termes de vacances et de tourisme, que l'on peut envisager le développement du tourisme social et la mise en œuvre de mesures sociales susceptibles de répondre à cette demande. En outre, le tourisme social peut faire partie de l'arsenal des moyens requis pour consolider une classe moyenne dans plusieurs pays.

2. Le développement d'un tourisme domestique moins dépendant des aléas extérieurs : le tourisme social est intimement lié au tourisme interne ou domestique qui, tout en se préoccupant de répondre aux besoins de délassement des populations résidantes, diminue la dépendance du tourisme national à l'égard des marchés extérieurs étrangers.

3. La clientèle des jeunes comme axe prioritaire: pour des pays africains dont la population est majoritairement jeune, cet axe est à privilégier avec des objectifs d'éducation (les voyages ne forment-ils pas la jeunesse ?), mais aussi pour créer un marché interne à long terme.

4. La sauvegarde du patrimoine naturel et culturel : sur ce plan, le tourisme social peut être un partenaire de premier plan, tant par son souci d'accessibilité et sa recherche de la qualité dans la relation entre les visiteurs et les visités qu'en raison du contenu culturel de l'expérience touristique.

5. Le développement local : à une échelle humaine, les équipements de tourisme social sont souvent réalisés en dehors des circuits traditionnels et représentent un espoir pour des économies fragiles.

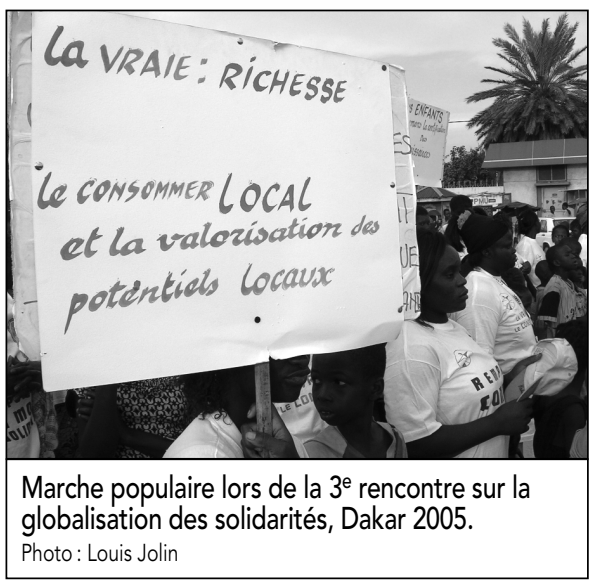


En outre, un approfondissement de la notion d'accessibilité a amené les auteurs de cette partie de l'étude à affirmer que le tourisme ne doit pas être accessible seulement aux visiteurs, mais aussi aux populations des territoires d'accueil qui doivent avoir accès à leurs propres ressources touristiques ainsi qu'aux bénéfices du tourisme, ce qui conduit naturellement à la notion de tourisme solidaire.

Les avis des experts africains consultés ont fait aussi ressortir les éléments suivants :

- la pluralité des conceptions de ce que peut être le tourisme social et le tourisme solidaire en Afrique ;

- la difficulté d'amalgamer la tradition et la modernité ;

- la prédominance des jeunes dans le continent africain et leurs attentes spécifiques en matière d'activités récréatives ;

- les contraintes liées à la pauvreté (souvent extrême) d'une majorité des populations du continent ;

- les dimensions socioculturelles de la problématique de l'accès au tourisme qui ne se résument pas aux seuls problèmes économiques des populations africaines;

- le poids de l'imaginaire et du sacré qui commande aux rites célébrant les pratiques liées à ce type de tourisme...

En conclusion préliminaire, les auteurs de la partie conceptuelle affirment qu'il serait avantageux pour les pays africains de s'engager, conformément aux attentes de leurs populations, sur la voie de la promotion du tourisme social pour au moins trois raisons :

- démocratiser les vacances et la pratique du tourisme, compte tenu des bénéfices de tous ordres que les participants peuvent en tirer ;

- créer un marché interne moins dépendant de l'extérieur ;

- contribuer au renforcement de la classe moyenne.

Ce bref résumé de la partie conceptuelle laisse un certain nombre de questions en suspens. Dans quelle mesure les gouvernements africains jouent-ils actuellement un rôle dans le développement du tourisme social et du tourisme solidaire dans leur pays? Dans quelle mesure les acteurs sur le terrain, que sont les associations de tourisme et les ONG, ont-ils mis en place des initiatives intéressantes qui bénéficient aux populations locales tout en favorisant une véritable relation entre les visiteurs et les visités? Ces deux questions ont fait l'objet des deux autres volets de l'étude.

\section{Des actions significatives mais insuffisantes de la part des administrations nationales publiques}

Le but de l'enquête effectuée auprès des administrations nationales du tourisme (ANT) africaines visait à recueillir de l'information sur le tourisme interne, sur les politiques et les programmes en matière de tourisme social et sur le soutien à des projets locaux de tourisme solidaire.

Un questionnaire a été envoyé aux ANT de 51 pays d'Afrique. Le tiers des pays avaient déjà répondu au moment de présenter le rapport préliminaire en novembre 2005 à Dakar lors de la $16^{e}$ session de l'Assemblée générale de l'OMT. À la suite d'une relance effectuée en 2006, de nouvelles réponses se sont ajoutées pour un total de 30 pays répondants. L'analyse présentée porte donc sur 30 questionnaires complétés par 18 pays francophones, 8 pays anglophones et 4 pays lusophones. II s'agit certes d'un échantillon assez représentatif qui permet d'avoir un bon aperçu du rôle et des actions des pouvoirs publics dans les secteurs du tourisme social et du tourisme solidaire et de dégager un certain nombre de conclusions et de recommandations.

Le questionnaire, qui comprenait 33 questions, dont 22 questions fermées, se divisait en trois grandes parties:

1. développement et promotion du tourisme interne (12 questions) ;

2. rôle des autorités gouvernementales dans le secteur du tourisme social (15 questions) ;

3. appui au développement du tourisme solidaire (6 questions).

L'analyse des résultats de cette enquête permet d'identifier quelques grandes tendances liées aux trois aspects étudiés.

Le tourisme interne occupe une place bien réelle sur le plan des objectifs et des stratégies de la politique nationale du tourisme en Afrique. II est à la fois utilisé comme outil économique visant à créer des emplois et à stimuler la demande nationale, comme outil social pour favoriser l'accès au voyage de divers segments de la population et comme outil politique en faveur de la paix et de l'intégration nationale.

\section{Tableau 1}

Liste des pays ayant répondu à l'enquête auprès des administrations nationales du tourisme

\begin{tabular}{l|l}
\hline Afrique du Sud & Maroc \\
Algérie & Mauritanie \\
Angola & Mozambique \\
Bénin & Niger \\
Burkina Faso & République centrafricaine \\
Cameroun & Rwanda \\
Congo Brazzaville & São Tomé et Príncipe \\
Érythrée & Sénégal \\
Éthiopie & Seychelles \\
Gabon & Soudan \\
Gambie & Swaziland \\
Guinée & Tanzanie \\
Guinée-Bissau & Tchad \\
Madagascar & Togo \\
Mali & Tunisie \\
\hline
\end{tabular}

Source : auteurs.

Le tourisme interne, qui connait un certain niveau de croissance dans plusieurs pays, s'adresse surtout aux familles et aux jeunes dont les principales motivations de voyage sont liées à la visite de parents et d'amis, à des fêtes et, dans une moindre mesure, à des événements sportifs ou à des voyages scolaires. Sa pratique, concentrée sur de courtes périodes de l'année, est quant à elle fortement caractérisée par l'usage de moyens de transport terrestre - autocar et automobile - et de l'hébergement chez des parents et des amis.

Du côté du tourisme social, un peu plus de la moitié des ANT ayant répondu à l'enquête affirment qu'il est pris en compte dans la politique nationale du tourisme. II s'agit d'une donnée qui peut surprendre, compte tenu des priorités de plusieurs gouvernements africains en matière de développement. Malgré les divers problèmes auxquels l'Afrique est confrontée, il semble que l'accès aux vacances et au tourisme pour le plus grand nombre ne soit pas absent du programme politique, même si sa mise en pratique est encore embryonnaire.

Les objectifs visés par le tourisme social sont similaires à ceux du tourisme interne et se traduisent par des initiatives associées au développement et à la diversification de l'offre ainsi que par des actions visant à faciliter directement le départ en vacances de la population. La diversité des acteurs identifiés au tourisme social révèle aussi l'importance relative d'administrations publiques autres que celle du tourisme et d'un réseau d'organismes, principalement du côté de la société civile. 
Si les taux de départ en vacances sont pratiquement inconnus, bien que certains indices puissent indiquer un taux qui pourrait varier de 15 à $30 \%$, selon les pays, les freins au développement du tourisme social sont quant à eux bien identifiés: le manque de ressources financières, l'absence d'une culture du voyage et des vacances et le manque d'information. Si le premier facteur ne constitue pas en soi une véritable surprise, force est de constater que les deux autres éléments doivent notamment interpeller les autorités en charge des programmes éducatifs.

En ce qui a trait au tourisme solidaire, il s'agit d'une réalité bien connue - les nombreux exemples cités en font preuve - qui bénéficie aussi de l'appui des ANT ou d'autres organismes publics. Ces appuis, qui vont de l'assistance technique à la commercialisation en passant par la formation, le renforcement des capacités et l'appui à la recherche de financement, ont pour but principal le développement économique des communautés locales, la participation des communautés au développement du tourisme, la réduction de la pauvreté, le développement durable du tourisme en régions et la diversification du produit touristique.

Le tourisme solidaire ou communautaire (community-based tourism) semble donc trouver un plus grand nombre d'applications que le tourisme social, même si, dans certains cas, les initiatives mises de l'avant peuvent concerner les mêmes clientèles. Cela peut sans doute s'expliquer par le fait que, à la base du concept de tourisme solidaire, le développement économique est davantage pris en compte.

S'appuyant sur ces éléments, les chercheurs concluent de la manière suivante:

- La pratique du tourisme interne demeure limitée sur le continent africain, même si elle peut constituer un élément intrinsèque de la politique nationale du tourisme.

- Le tourisme social est pris en compte par plusieurs ANT, malgré la faiblesse relative des stratégies, des initiatives et des moyens mis en œuvre.

- Le taux de départ en vacances des populations africaines est très bas, à cause principalement de problèmes financiers, mais aussi dû à une relative absence d'une culture du voyage et au manque d'information.
- Le tourisme solidaire est un secteur connu et qui intéresse les pouvoirs publics comme une alternative au développement économique des communautés locales.

\section{Une multiplicité d'approches et une communauté d'objectifs pour développer le tourisme solidaire}

Dans cette troisième partie de l'étude, la parole est donnée aux acteurs de terrain pour identifier les points communs entre les différentes structures, leur approche respective et leurs objectifs.

Les chercheurs ont envoyé un questionnaire à 134 organismes dans 31 pays africains. Sur les 50 organismes qui ont répondu, représentant 21 pays, 42 (19 pays) répondaient réellement aux critères de tourisme solidaire.

Avec seulement 42 réponses étudiées, la recherche ne peut être considérée comme exhaustive. Elle donne simplement à voir la réalité instantanée d'un certain nombre d'organismes africains qui expérimentent et développent, avec plus ou moins de réussite, un tourisme différent.

Si les exemples repérés lors de l'enquête ont pu laisser entrevoir une multiplicité d'approches et de solutions pour bâtir et mettre en œuvre un projet de tourisme solidaire, les chercheurs ont mis à jour une communauté d'objectifs partagés par toutes ces organisations:

- la nécessité de développer une forme de tourisme respectueux de la population et de l'environnement ;

- la reconnaissance des populations locales comme étant parties prenantes du développement économique ;

- l'éradication de la pauvreté et le développement de l'autonomie économique et de l'éducation;

- la volonté de capitaliser sur leur patrimoine culturel et naturel, leurs seuls biens.

Les acteurs partagent également une forte détermination, car tout concourt à rendre difficile la réalisation des divers projets: le manque d'éducation et de formation des acteurs euxmêmes et/ou des populations locales; le manque d'argent, de moyens, d'infrastructures, de volonté politique; des environnements naturel, social et politique critiques.

\section{Tableau 2}

Liste des pays représentés à l'enquête auprès des acteurs locaux

\begin{tabular}{l|l}
\hline Afrique du Sud & Mali \\
Bénin & Maroc \\
Burkina Faso & Mauritanie \\
Cameroun & Namibie \\
Congo (RDC) & Ouganda \\
Égypte & Sénégal \\
Guinée & Swaziland \\
Guinée-Bissau & Tanzanie \\
Kenya & Togo \\
Madagascar & \\
\hline Source: auteurs. & \\
\hline
\end{tabular}

Les expériences de l'Afrique du Sud avec le label FITSA (Fair Trade in Tourism South Africa), de Madagascar avec l'ONG Fanamby, et de la Namibie avec l'association Nacobta, montrent l'importance de se fédérer au sein d'un réseau. Le Sénégal s'ouvre à cette démarche avec la création de R.A.T.RE.S DEGGO, un réseau national d'associations pour le tourisme responsable au Sénégal.

L'existence d'un réseau non seulement peut renforcer le poids de ces structures auprès des autorités locales, mais elle facilite la négociation auprès des partenaires étrangers, notamment les voyagistes.

Le tourisme solidaire, qui a pour objectif la lutte contre la pauvreté, butte à un moment donné sur le problème de l'éducation et de la formation des populations locales. Sans aides et sans stratégies des pouvoirs publics, la situation semble complexe et difficile à résoudre.

Beaucoup d'organisations font par ailleurs part de leur souci de ne pas baser leur économie uniquement sur leur activité touristique, mais ce n'est pas toujours possible de faire autrement.

Il faut aussi noter que beaucoup d'organismes bénéficient directement ou indirectement de l'outil Internet pour leur promotion et que la majorité de ceux-ci sont conscients de l'importance de ce vecteur de communication.

En outre, un grand nombre de structures d'accueil sont connues par le bouche-à-oreille, ce qui est la base des activités de service. Les outils de communication sous forme de publication sont plus rares, car ils sont évidemment plus coûteux. En dehors de ces outils de communication, les structures attendent beaucoup de la mise en relation avec les partenaires étrangers, notamment européens. 
La plupart des organismes insistent sur la nécessité de créer des partenariats avec des structures et des voyagistes qui partagent la même éthique, conscients de la possibilité de vite verser dans la publicité et la communication du tourisme de masse.

Par ailleurs, plusieurs pays ont des difficultés à instaurer la pratique du tourisme domestique. Quelques pays y arrivent, comme l'Afrique du Sud, parce qu'une partie de la population est économiquement plus aisée.

Il est difficile d'évaluer concrètement et précisément l'implication des populations locales dans toutes les phases du projet. Tous les organismes cependant déclarent avoir monté leur projet avec des structures et des personnes locales. Deux certitudes demeurent:

- La population locale est impliquée en tant qu'actrice de l'activité touristique: emplois de personnes locales, quel que soit le métier, importance du logement chez l'habitant ou au sein de la communauté, rencontres organisées avec les populations locales.

- La population locale est impliquée dans le choix des projets collectifs mis en œuvre en partie grâce aux revenus de l'activité touristique.

\section{Conclusions générales et quelques recommandations}

Les trois volets de l'étude sont complémentaires et révèlent non seulement un réel potentiel de développement du tourisme social et du tourisme solidaire sur le continent africain, mais aussi des réalisations intéressantes des pouvoirs publics comme des acteurs actifs sur le terrain.

Menés en parallèle, les trois volets font cependant ressortir quelques différences dans l'appréciation de la réalité actuelle et du potentiel de développement du tourisme social et du tourisme solidaire. Les experts consultés soulignent les obstacles d'ordres économique et culturel, tout en identifiant des repères significatifs pour l'essor du tourisme social en Afrique, tandis que les administrations nationales du tourisme mettent plutôt en lumière des actions et des expériences qui vont dans le sens d'un appui au tourisme social et au tourisme solidaire. Enfin, les acteurs associatifs engagés dans des activités de tourisme solidaire insistent sur les retombées positives de leur action, en faisant état aussi des difficultés rencontrées.

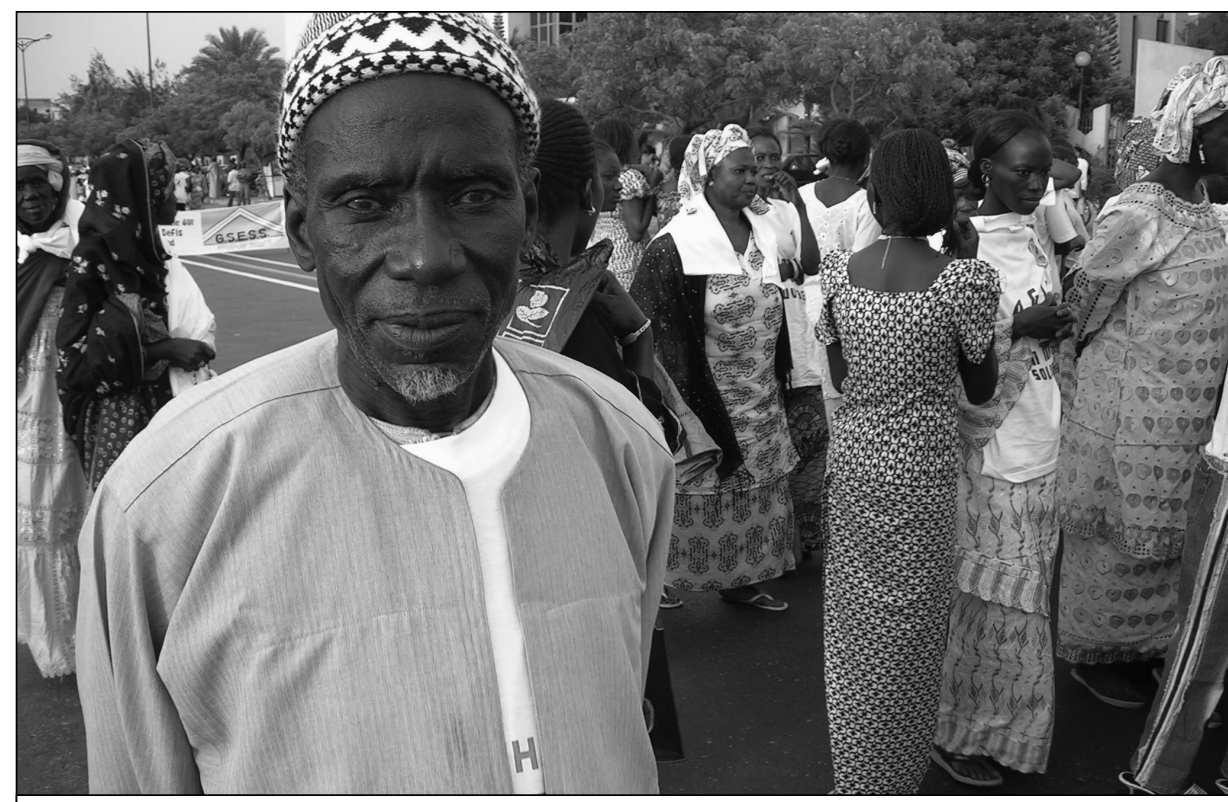

Le respect des populations locales au coeur d'une stratégie de développement durable. Photo : Louis Jolin

Cette étude n'est pas exhaustive, loin s'en faut, bien qu'un nombre relativement important d'administrations nationales et d'associations aient accepté de participer aux enquêtes.

L'Afrique est un vaste continent et les conditions socioéconomiques de même que les contextes politiques des divers pays sont loin d'être homogènes. Toute synthèse est périlleuse et le danger est réel d'effacer les disparités entre les pays. II se dégage néanmoins des orientations générales, comme l'ont révélé les conclusions des divers volets de l'étude:

- l'intérêt de développer un tourisme interne (domestique) fort, moins dépendant des marchés extérieurs ;

- la démocratisation progressive des vacances et du tourisme peut apporter des bénéfices aux populations, dans le respect des priorités de développement des pays;

- une attention toute particulière accordée aux jeunes et aux familles est plus que souhaitable ;

- la prise en compte des dimensions socioculturelles de l'accès au tourisme ne se résume pas aux seuls problèmes économiques des populations africaines ;

- le renforcement de la classe moyenne dans de nombreux pays passe par des mesures sociales bien définies dans plusieurs secteurs, y compris ceux du tourisme et des vacances ;
- la préservation et la mise en valeur du patrimoine naturel et culturel et l'augmentation des bénéfices pour les populations qui accueillent les visiteurs sont compatibles avec l'essor du tourisme social et du tourisme solidaire ;

- la conciliation du développement touristique, de la protection de l'environnement et du respect des populations locales s'inscrivent dans une stratégie de développement local durable;

- un appui aux entreprises d'économie sociale et solidaire, que sont principalement les associations et les coopératives, est une voie à privilégier.

Les conclusions s'adressent en premier lieu au Bureau international du tourisme social et elles sauront alimenter les débats au sein de la section Afrique qu'il a mise sur pied en 2004. Le BITS est disposé, dans la mesure du possible, à apporter son soutien et son expertise aux pays prêts à s'engager dans la voie d'un développement du tourisme social et du tourisme solidaire, en collaboration avec les autorités locales et les collectivités territoriales. Ce soutien pourrait se concrétiser par l'assistance technique (montage de projets) et par la formation professionnelle. Pour ce faire, le BITS compte aussi sur l'aide d'organismes internationaux (OMT, $\mathrm{PNUD}^{7}, \mathrm{UNESCO}^{8}$ et diverses organisations non gouvernementales). 
Les associations et les coopératives ont contribué par le monde à développer des entreprises de tourisme social et de tourisme solidaire et le BITS, qui regroupe plusieurs d'entre elles, est un partenaire de premier plan de l'économie sociale et solidaire.

Les organisations spécialisées dans le tourisme social et le tourisme solidaire ont, grâce à leur réseau de membres, des moyens humains pour aider à la formation et à la sensibilisation dans ces secteurs.

Afin de donner suite à la présente étude et aux orientations qui en découlent, les recommandations suivantes sont formulées par les responsables de l'étude:

- soutenir la création d'un réseau africain des opérateurs et des acteurs du tourisme social et du tourisme solidaire ;

- créer les outils de communication au sein ou en faveur de ce réseau : un annuaire; un site Internet spécifique, un guide des bonnes pratiques;

- organiser tous les deux ans un séminaire africain sur les thèmes du tourisme social et du tourisme solidaire (le $3^{e}$ Forum international du tourisme solidaire devant d'ailleurs se tenir en Afrique en 2008) ;

- envisager une étude plus précise sur les dispositifs nationaux et interrégionaux permettant de développer le tourisme social à l'intérieur des pays africains ;

- mettre sur pied des modules de formation souples et facilement transposables d'un pays à l'autre pour les acteurs du tourisme social en utilisant notamment les technologies de l'information (CD-ROM, Internet...).

Charles-Étienne Bélanger est directeur du Bureau international du tourisme social, dont le siège social est situé à Bruxelles.

Louis Jolin est professeur au Département d'études urbaines et touristiques à l'École des sciences de la gestion de l'Université du Québec à Montréal et responsable du comité scientifique du BITS.

Jean-Marc Mignon est délégué général de l'Union nationale des associations de tourisme et de plein air (UNAT - France) et vice-président du BITS.

\section{Notes}

1 Statistiques officielles de l'OMT (2006).

2 L'événement TourismAfrica a réuni du 10 au 15 septembre 2006 à Genève des décisionnaires des secteurs public et privé impliqués dans le développement de l'industrie du tourisme africain ainsi que des représentants d'institutions internationales actifs dans ce domaine.

3 La section Afrique du BITS a été créée à l'occasion du Congrès mondial du tourisme social tenu à Blankenberge (Belgique) du 28 au 30 avril 2004.

4 Le texte été rédigé par Louis Jolin, responsable du comité scientifique du BITS et professeur à l'École des sciences de la gestion de l'Université du Québec à Montréal (ESG UQÀM) (Canada) et Mimoun Hillali, membre du comité scientifique du BITS et professeur à l'Institut supérieur de tourisme de Tanger (Maroc).

Les auteurs remercient de leurs avis constructifs les experts suivants: Hussein Sosovele (Institute of Resource Assessment, Université Dar es Salaam, Tanzanie); Kwaku A. Boakye (Department of Geography and Tourism, University of Cape Coast, Ghana) ; Modibo Kire (Département de géographie, Flash, Bamako, Mali) ; El Hadj Malick Mbaye (Bureau du tourisme, ambassade du Sénégal à Paris, Sénégal).

Les auteurs remercient aussi de leurs commentaires Bruno Sarrasin, professeur à I'ESG UQÀM, membre du comité scientifique du BITS, et deux de ses étudiants à la maitrise en gestion et planification du tourisme, Mehdi Driss (Tunisie) et Arnaud Duthuillé (France), ainsi que, pour son appui au traitement de la documentation, Karine Boulet-Gaudreault (Québec).

5 Cette partie de l'étude a bénéficié de l'appui financier du ministère français des Affaires étrangères et des conseils du réseau italien AITR (Associazione Italiana Turismo Responsabile).

6 Le tourisme social se réfère à «l'ensemble des rapports et des phénomènes résultant de la participation au tourisme des couches sociales aux revenus modestes. Cette participation est rendue possible ou facilitée par des mesures d'un caractère social bien défini. » (Statuts du BITS) De manière plus concrète, le tourisme social "se réfère aux programmes, aux réalisations et aux actions qui visent à rendre effectifs le droit aux vacances et l'accessibilité au tourisme à tous les groupes de population, notamment les jeunes, les familles, les retraités, les personnes aux revenus modestes, les personnes à capacité physique restreinte, mais qui visent aussi la qualité de la relation entre les visiteurs et les communautés d'accueil. » (Jolin, 2004:6) On pense, entre autres, aux colonies de vacan- ces pour les plus jeunes, aux villages-vacances pour les familles, aux auberges de jeunesse, aux gîtes ruraux, aux voyages éducatifs et culturels pour les jeunes ou pour les aînés. II existe aussi des formes traditionnelles de loisir et de convivialité en Afrique qui peuvent relever du tourisme social, version locale: moussems, pèlerinages, fêtes d'initiation, fêtes religieuses ou foraines...

7 UNESCO : Organisation des Nations Unies pour l'éducation, la science et la culture.

8 PNUD : Programme des Nations Unies pour le développement.

\section{Bibliographie}

BITS (1996), "Pour une vision humaniste et sociale du tourisme ", Déclaration de Montréal, Congrès du BITS, septembre 1996, Bruxelles, Bureau international du tourisme social.

BITS, Statuts, adoptés en 1963 et modifiés à quelques reprises, la dernière modification remontant à mai 2002, Bruxelles, Bureau international du tourisme social.

BITS, [www.bits-int.org], (consulté pendant la recherche).

El Alaoui, Françoise (1999), Le tourisme équitable, mémoire de master, Paris, École supérieure de gestion.

Haulot, Arthur (1980), Tourisme social, phénomène universel. Caractéristiques sociologiques et culturelles, communication au Congrès du BITS.

Hillali, Mimoun (1998), «Saidia (Maroc oriental) : une station balnéaire à la recherche de son identité touristique ", Le tourisme au Maghreb, diversification du produit et développement local et régional, Actes du $5^{\mathrm{e}}$ colloque Maroc allemand, Tanger, p. 164-171.

Jolin, Louis (2004), "Le tourisme social, un concept riche de ses évolutions ", Le tourisme social dans le monde (Bits information), no 141, p. 6-8.

Matteudi, Guy (1997), Les vacances, facteur d'insertion sociale, Rapport présenté au Conseil national du tourisme, Paris.

OMT (2004), Le tourisme et la réduction de la pauvreté. Recommandations pour l'action, Madrid, Organisation mondiale du tourisme.

OMT (2006), Faits saillants du tourisme international (tour d'horizon du tourisme international en 2005), Madrid, Organisation mondiale du tourisme.

Sidi Boumedine, Rachid, et Laure Veirier (2003), Le Sahara des cultures et des peuples - Vers une stratégie pour un développement durable du tourisme au Sahara dans une perspective de lutte contre la pauvreté, Paris, UNESCO.

UNAT (2005), Tourisme solidaire. Des voyages vers l'essentiel, Paris, Union nationale des associations de tourisme et de plein air. 